\title{
NOTE ON THE EXPANSION OF A POWER SERIES INTO A CONTINUED FRACTION
}

\author{
H. S. WALL
}

1. Introduction. In view of the fact that the continued fraction frequently furnishes a method for summing a slowly convergent or even divergent power series, it is desirable to have a simple algorithem for obtaining the continued fraction. We present here such an algorithm based upon the fact that the process for constructing a sequence of orthogonal polynomials can be so arranged that it gives simultaneously a continued fraction expansion for a power series. It has been known at least since Tschebycheff that the problem of constructing a sequence of orthogonal polynomials is related to the problem of expanding a power series into a continued fraction. However, the fact that the two problems are actually identical does not seem to have been emphasized.

2. The expansion of a power series into a $J$-fraction. A continued fraction of the form

$$
\frac{a_{0}}{b_{1}+z}-\frac{a_{1}}{b_{2}+z}-\frac{a_{2}}{b_{3}+z}-\cdots
$$

is called a $J$-fraction. The $a_{p}$ and $b_{p}$ are constants, and $z$ is a complex variable. We shall suppose that the $a_{p}$ are different from zero. We denote by $A_{p}(z)$ and $B_{p}(z)$ the $p$ th numerator and denominator, respectively, of the $J$-fraction, so that $A_{p}(z) / B_{p}(z)$ is its $p$ th approximant. The usual recurrence formulas

$$
\begin{gathered}
A_{0}=0, A_{1}=a_{0}, \quad A_{p}=\left(b_{p}+z\right) A_{p-1}-a_{p-1} A_{p-2}, \\
\quad p=2,3,4, \cdots, \\
B_{0}=1, B_{1}=b_{1}+z, B_{p}=\left(b_{p}+z\right) B_{p-1}-a_{p-1} B_{p-2},
\end{gathered}
$$

show that $A_{p}(z)$ is a polynomial of degree $p-1$, and $B_{p}(z)$ is a polynomial of degree $p$ :

$$
\begin{aligned}
& A_{p}(z)=\alpha_{p, 0} z^{p-1}+\alpha_{p, 1} z^{p-2}+\cdots+\alpha_{p, p-1}, \\
& B_{p}(z)=\beta_{p, 0} z^{p}+\beta_{p, 1} z^{p-1}+\cdots+\beta_{p, p} .
\end{aligned}
$$

We note that

Presented to the Society, August 14, 1944; received by the editors February 21, 1944, and, in revised form, June 5, 1944. 


$$
\beta_{p, 0}=1, \quad \beta_{p, 1}=b_{1}+b_{2}+\cdots+b_{p} .
$$

By means of (2.2) we readily obtain the determinant formula

$$
\begin{aligned}
A_{p}(z) B_{p-1}(z)-A_{p-1}(z) B_{p}(z)=a_{0} a_{1} \cdots & a_{p-1}, \\
& p=1,2,3, \cdots .
\end{aligned}
$$

Consequently we find, with the aid of (2.4), that

$$
\frac{A_{n+1}(z)}{B_{n+1}(z)}-\frac{A_{n}(z)}{B_{n}(z)}=\frac{a_{0} a_{1} \cdots a_{n}}{z^{2 n+1}}+\frac{h_{n}}{z^{2 n+2}}+\cdots
$$

where

$$
h_{n}=-a_{0} a_{1} \cdots a_{n}\left(b_{1}+b_{2}+\cdots+b_{n+1}\right) .
$$

It follows that there exists a power series

$$
P(1 / z)=c_{0} / z+c_{1} / z^{2}+c_{2} / z^{3}+\cdots
$$

such that the expansion in descending powers of $z$ of $A_{n}(z) / B_{n}(z)$ agrees term by term with $P(1 / z)$ for the first $2 n$ terms $(n=1,2$, $3, \cdots)$. This uniquely determined power series is called the equivalent power series of the $J$-fraction.

We shall now write down formulas connecting the various constants, $\alpha_{p, q}, \beta_{p, q}, c_{p}, a_{p}$ and $b_{p}$. These formulas serve as an algorithm for expanding a given power series $P(1 / z)$ into a $J$-fraction, and, conversely, for obtaining the equivalent power series of a given $J$-fraction.

$$
\begin{aligned}
& \beta_{00}=1, \quad c_{0} \beta_{00}=a_{0}, \quad c_{1} \beta_{00}=h_{0}=-a_{0} b_{1} \\
& b_{1}=-h_{0} / a_{0}, \quad\left(\beta_{10}, \beta_{11}\right)=\left(1, b_{1}\right) \\
& \left(c_{2}, c_{1}\right)\left(\begin{array}{c}
\beta_{10} \\
\beta_{11}
\end{array}\right)=a_{0} a_{1}, \quad\left(c_{3}, c_{2}\right)\left(\begin{array}{c}
\beta_{10} \\
\beta_{11}
\end{array}\right)=h_{1}=-a_{0} a_{1}\left(b_{1}+b_{2}\right) \\
& b_{2}=h_{0} / a_{0}-h_{1} / a_{0} a_{1}, \\
& \left(\beta_{20}, \beta_{21}, \beta_{22}\right)=\left(\beta_{10}, \beta_{11}\right)\left(\begin{array}{l}
1, b_{2}, 0 \\
0,1, b_{2}
\end{array}\right)-a_{1}\left(0,0, \beta_{00}\right) \\
& \left(c_{4}, c_{3}, c_{2}\right)\left(\begin{array}{l}
\beta_{20} \\
\beta_{21} \\
\beta_{22}
\end{array}\right)=a_{0} a_{1} a_{2}, \\
& \left(c_{5}, c_{4}, c_{3}\right)\left(\begin{array}{l}
\beta_{20} \\
\beta_{21} \\
\beta_{22}
\end{array}\right)=h_{2}=-a_{0} a_{1} a_{2}\left(b_{1}+b_{2}+b_{3}\right)
\end{aligned}
$$




$$
\begin{aligned}
& b_{3}=h_{1} / a_{0} a_{1}-h_{2} / a_{0} a_{1} a_{2}, \\
& \left(\beta_{30}, \beta_{31}, \beta_{32}, \beta_{33}\right)=\left(\beta_{20}, \beta_{21}, \beta_{22}\right)\left(\begin{array}{llll}
1, & b_{3}, & 0, & 0 \\
0, & 1, & b_{3}, & 0 \\
0, & 0, & 1, & b_{3}
\end{array}\right) \\
& -a_{2}\left(0,0, \beta_{10}, \beta_{11}\right) \text {, }
\end{aligned}
$$

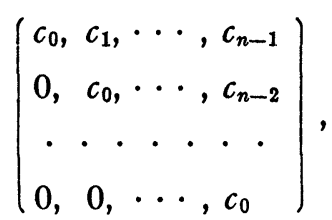

$$
\begin{aligned}
& n=1,2,3, \cdots,\left(\beta_{n, 0}=1\right) .
\end{aligned}
$$

By way of illustration, we shall obtain the third approximant of the $J$-fraction for the function $P(1 / z)=\log (1+1 / z)$. Here $c_{p}$ $=(-1)^{p} /(p+1), p=0,1,2, \cdots$. We then have:

$$
\begin{aligned}
& \beta_{00}=1, \quad c_{0}=a_{0}=1 \text {; } \\
& b_{1}=1 / 2, \quad\left(\beta_{10}, \beta_{11}\right)=(1,1 / 2) \text {, } \\
& (1 / 3,-1 / 2)\left(\begin{array}{c}
1 \\
1 / 2
\end{array}\right)=1 / 12=a_{1} \text {, } \\
& (-1 / 4,1 / 3)\left(\begin{array}{c}
1 \\
1 / 2
\end{array}\right)=-1 / 15=h_{1} \text {; } \\
& b_{2}=1 / 2, \quad\left(\beta_{20}, \beta_{21}, \beta_{22}\right)=(1,1 / 2)\left(\begin{array}{ccc}
1, & 1 / 2, & 0 \\
0, & 1, & 1 / 2
\end{array}\right)-(1 / 12)(1,0,1) \\
& =(1,1,1 / 6) \text {, } \\
& (1 / 5,-1 / 4,1 / 3)\left(\begin{array}{c}
1 \\
1 \\
1 / 6
\end{array}\right)=1 / 180=a_{0} a_{1} a_{2}, \quad a_{2}=1 / 15 \\
& (-1 / 6,1 / 5,1 / 4)\left(\begin{array}{c}
1 \\
1 \\
1 / 6
\end{array}\right)=-1 / 120=h_{2} \\
& b_{3}=1 / 2, \quad\left(\beta_{30}, \beta_{31}, \beta_{32}, \beta_{33}\right)=(1,3 / 2,3 / 5,1 / 20) \text {; } \\
& \left(\alpha_{30}, \alpha_{31}, \alpha_{32}\right)=(1,1,11 / 60) \text {. }
\end{aligned}
$$


Consequently, the third approximant of the $J$-fraction is

$$
\begin{aligned}
\frac{A_{8}(z)}{B_{3}(z)} & =\frac{1}{1 / 2+z}-\frac{1 / 12}{1 / 2+z}-\frac{1 / 15}{1 / 2+z} \\
& =\frac{z^{2}+z+(11 / 60)}{z^{3}+(3 / 2) z^{2}+(3 / 5) z+(1 / 20)} .
\end{aligned}
$$

We remark that for $z=1$ this gives $\log 2=.69312 \cdots$, which is exact to four decimal places. Only six coefficients of the power series were used in the computation.

By the same method we find that the seventh approximant of the $J$-fraction expansion of the divergent power series

$$
\frac{B_{1}}{1 \cdot 2 \cdot z}-\frac{B_{3}}{3 \cdot 4 \cdot z^{3}}+\frac{B_{5}}{5 \cdot 6 \cdot z^{5}}-\cdots,
$$

where $B_{1}=1 / 6, B_{3}=1 / 30, B_{5}=1 / 42, \cdots$ are the Bernoulli numbers, is

$$
\begin{aligned}
\frac{1 / 12}{z} & +\frac{1 / 30}{z}+\frac{53 / 210}{z}+\frac{195 / 371}{z}+\frac{22999 / 22737}{z} \\
& +\frac{29944523 / 19733142}{z}+\frac{109535241009 / 48264275462}{z} .
\end{aligned}
$$

Stieltjes $[3, \text { p. 521 }]^{1}$ proved that this $J$-fraction converges for $R(z)>0$ to the remainder $J(z)$ in Stirling's formula $\log \Gamma(z)$ $=(z-1 / 2) \log z-z+(1 / 2) \log (2 \pi)+J(z)$. He remarked that the law of formation of the coefficients in the $J$-fraction seems to be extremely complicated.

3. Proof of the formulas (2.8) and (2.9). We shall first prove that the formulas (2.8) constitute an arrangement of the algorithm for constructing a sequence of polynomials $B_{n}(z)=z^{n}+\beta_{n, 1} z^{n-1}+\cdots$ $+\beta_{n, n}$ which are orthogonal relative to a certain operator $S$. We define $S$ to be the operator which replaces every $z^{p}$ by $c_{p}$ in any polynomial upon which it operates:

$$
\begin{aligned}
S\left(\beta_{0} z^{n}+\beta_{1} z^{n-1}+\cdots+\beta_{n}\right) & =S\left(\beta_{0} z^{n}+\beta_{1} z^{n-1}+\cdots+\beta_{n} z^{0}\right) \\
& =\beta_{0} c_{n}+\beta_{1} c_{n-1}+\cdots+\beta_{n} c_{0},
\end{aligned}
$$

where $c_{0}, c_{1}, \cdots, c_{p}, \cdots$ are given constants. Two polynomials $B_{p}$ and $B_{q}$ are said to be orthogonal if $S\left(B_{p} B_{q}\right)=0$ when the degrees $p$ and $q$ are unequal. We shall prove the following theorem:

1 Numbers in brackets refer to the bibliography at the end of the paper. 
Theorem A. Let $m$ be a positive integer, and put

$$
\Delta_{p}=\left|\begin{array}{lll}
c_{0}, c_{1}, & \cdots, c_{p} \\
c_{1}, c_{2}, & \cdots, c_{p+1} \\
\cdot . & . & \cdot . \cdot \\
c_{p}, c_{p+1}, & \cdots, c_{2 p}
\end{array}\right|, \quad p=0,1,2, \cdots .
$$

There exists a sequence of polynomials $B_{n}(z)=z^{n}+\beta_{n, 1} z^{n-1}+\cdots+\beta_{n, n}$, $n=0,1,2, \cdots, m$, such that

$$
S\left(B_{p} B_{q}\right)\left\{\begin{array}{lll}
=0 & \text { if } & p \neq q, p \leqq m, q \leqq m, \\
\neq 0 & \text { if } & p=q<m,
\end{array}\right.
$$

if and only if $\Delta_{p} \neq 0$ for $p=0,1,2, \cdots, m-1$. The polynomials are uniquely determined by the formulas

$$
\begin{array}{r}
B_{-1}=0, \quad B_{0}=1, \quad B_{p}=\left(b_{p}+z\right) B_{p-1}-a_{p-1} B_{p-2}, \\
p=1,2,3, \cdots, m,
\end{array}
$$

where

$$
\begin{aligned}
S\left(z^{p} B_{p}\right) & =a_{0} a_{1} \cdots a_{p} \neq 0, \\
S\left(z^{p+1} B_{p}\right) & =-a_{0} a_{1} \cdots a_{p}\left(b_{1}+b_{2}+\cdots+b_{p+1}\right), \\
p & =0,1,2, \cdots, m-1 .
\end{aligned}
$$

Proof. We suppose first that $\Delta_{p} \neq 0$ for $p=0,1, \cdots, m-1$, and shall prove that the required polynomials exist uniquely, and are given recurrently by (3.2) and (3.3). Since $B_{0}=1$, we have: $S\left(B_{0}^{2}\right)$ $=S(1)=S\left(z^{0}\right)=c_{0}=\Delta_{0} \neq 0$. Let $B_{1}=b_{1}+z$. Then, $S\left(B_{1}\right)=b_{1} c_{0}+c_{1}=0$ if and only if

$$
S\left(B_{0}\right)=a_{0}, \quad S\left(z B_{0}\right)=-a_{0} b_{1} .
$$

Using induction, suppose that $B_{0}, B_{1}, \cdots, B_{n}, n<m$, have been uniquely determined such that (3.1) holds for $p \leqq n, q \leqq n,(3.2)$ holds for $p \leqq n$, and (3.3) holds for $p \leqq n-1$. Now, an arbitrary polynomial of degree $n+1$ in which the coefficient of $z^{n+1}$ is unity can be expressed uniquely in the form $B_{n+1}=\left(z+b_{n+1}\right) B_{n}-a_{n} B_{n-1}+k_{0} B_{0}+k_{1} B_{1}+\ldots$ $+k_{n-2} B_{n-2}$, where $b_{n+1}, a_{n}, k_{0}, k_{1}, \cdots, k_{n-2}$ are suitable constants. The conditions $S\left(z^{p} B_{n+1}\right)=0, p=0,1, \cdots, n-2$, give in succession: $k_{0} a_{0}=0, k_{1} a_{0} a_{1}=0, \cdots, k_{n-2} a_{0} a_{1} \cdots a_{n-2}=0$, so that, since $a_{p} \neq 0$ for $p=0,1, \cdots, n-2$, we must have $k_{0}=k_{1}=\cdots=k_{n-2}=0$. From the conditions $S\left(z^{n-1} B_{n+1}\right)=0$ and $S\left(z^{n} B_{n+1}\right)=0$, we then find that $S\left(z^{n} B_{n}\right)=a_{0} a_{1} \cdots a_{n}$ and $S\left(z^{n+1} B_{n}\right)=-a_{0} a_{1} \cdots a_{n}\left(b_{1}+b_{2}+\cdots\right.$ $\left.+b_{n+1}\right)$. Then, from the system of equations: $S\left(z^{p} B_{n}\right)=0, p=0$, $1, \cdots, n-1, S\left(z^{n} B_{n}\right)=a_{0} a_{1} \cdots a_{n}$, we find at once that 


$$
\Delta_{n}=a_{0} a_{1} \cdots a_{n} \Delta_{n-1},
$$

and, inasmuch as $n<m$, we see that $a_{n} \neq 0$. Consequently, $B_{n+1}$ is uniquely determined, and (3.2), (3.3) hold for $p=n+1$ and $p=n$, respectively. Also, $S\left(B_{p} B_{q}\right)=0$ for $p \neq q, p \leqq n+1, q \leqq n+1$. Moreover, if $n+1<m$, then $S\left(B_{n+1}^{2}\right)=S\left(z^{n+1} B_{n+1}\right) \neq 0$, for otherwise we would have $\Delta_{n+1}=0$. We have proved that the condition $\Delta_{p} \neq 0$, $p=0,1, \cdots, m-1$, is sufficient for the polynomials to exist (uniquely) and satisfy the stated conditions.

Conversely, the condition is necessary. For, it is obviously necessary that $\Delta_{0}=c_{0} \neq 0$; and if $S\left(z^{p} B_{n}\right)=0$, for $p=0,1,2, \cdots, n-1$, $S\left(z^{n} B_{n}\right)=g_{n} \neq 0, n<m$, then the relation $\Delta_{n}=g_{n} \Delta_{n-1}$ must hold, and hence $\Delta_{p} \neq 0, p=0,1,2, \cdots, m-1$.

One will now readily see that the polynomials $B_{p}$ given by (3.2) and (3.3) are the same as those given by (2.8).

TheOREM B. Let $\Delta_{p} \neq 0, p=0,1,2, \cdots$, and define polynomials $A_{n}(z)=\alpha_{n, 0} z^{n-1}+\alpha_{n, 1} z^{n-2}+\cdots+\alpha_{n, n-1}$ by means of (2.9). Then,

(3.5) $\frac{A_{n}(z)}{B_{n}(z)}=\frac{a_{0}}{b_{1}+z}-\frac{a_{1}}{b_{2}+z}-\cdots-\frac{a_{n-1}}{b_{n}+z}, n=1,2,3, \cdots$,

and we have the formal power series identity

$$
P(1 / z) B_{n}(z)-A_{n}(z)=\frac{a_{0} a_{1} \cdots a_{n}}{z^{n+1}}+\frac{h_{n}}{z^{n+2}}+\cdots,
$$

where $h_{n}=-a_{0} a_{1} \cdots a_{n}\left(b_{1}+b_{2}+\cdots+b_{n+1}\right)$ and $P(1 / z)=\sum\left(c_{p} / z^{p+1}\right)$.

Proof. Let us define polynomials $A_{n}(z)$ by means of the formulas $A_{-1}=-1, A_{0}=0, A_{p}=\left(b_{p}+z\right) A_{p-1}-a_{p-1} A_{p-2}, p=1,2,3, \cdots$ From these recurrence formulas and (3.2) it follows that (3.5) holds. Furthermore, we may conclude from the determinant formula (2.5) that there exists a power series $P^{*}(1 / z)=\sum\left(c_{p}^{*} / z^{p+1}\right)$ such that

$$
P^{*}(1 / z) B_{n}(z)-A_{n}(z)=\frac{a_{0} a_{1} \cdots a_{n}}{z^{n+1}}+\frac{h_{n}}{z^{n+2}}+\cdots
$$

On equating coefficients of corresponding powers of $z$ on either side of this identity we find that precisely the relations (3.3) hold but with $c_{p}$ replaced by $c_{p}^{*}$. Inasmuch as those relations determine the $c_{p}$ uniquely in terms of the $a_{p}$ and $b_{p}$, we conclude that $c_{p}^{*}=c_{p}$, $p=0,1,2, \cdots$, or $P^{*}(1 / z)=P(1 / z)$, so that (3.6) holds. The relation (2.9) may now be obtained by equating the coefficients of $z^{0}, z^{1}, \cdots, z^{n-1}$ on either side of the identity (3.6). 
This completes the proof of the formulas (2.8) and (2.9) connecting the constants $\alpha_{p, q}, \beta_{p, q}, c_{p}, a_{p}, b_{p}$ of a $J$-fraction and its equivalent power series.

4. The expansion of a power series into an $S$-fraction. If we replace $z$ by $1 / z$ in the power series (2.7) and in its $J$-fraction expansion (2.1), the series becomes

$$
P(z)=c_{0} z+c_{1} z^{2}+c_{2} z^{3}+\cdots,
$$

and the $J$-fraction becomes

$$
\frac{a_{0} z}{1+b_{1} z}-\frac{a_{1} z^{2}}{1+b_{2} z}-\frac{a_{2} z^{2}}{1+b_{3} z}-\ldots .
$$

An important special case arises when all the $b_{p}$ are equal to zero. For, in this case it is evident that $P(z) / z$ contains only even powers of $z$. If we change the notation and replace $c_{2 n}$ by $c_{n}$, we see that the power series

$$
c_{0} z+0 z^{2}+c_{1} z^{3}+0 z^{4}+c_{2} z^{5}+\cdots
$$

has the expansion

$$
\frac{a_{0} z}{1}-\frac{a_{1} z^{2}}{1}-\frac{a_{2} z^{2}}{1}-\ldots
$$

Let us now remove a factor $z$ from both (4.3) and (4.4), and subsequently replace $z^{2}$ by $z$. Afterwards, we again multiply both the series and continued fraction by $z$ and then replace $z$ by $1 / z$. The series then becomes

$$
\frac{c_{0}}{z}+\frac{c_{1}}{z^{2}}+\frac{c_{2}}{z^{3}}+\cdots,
$$

and the continued fraction becomes

$$
\frac{a_{0}}{z}-\frac{a_{1}}{1}-\frac{a_{2}}{z}-\frac{a_{3}}{1}-\frac{a_{4}}{z}-\ldots
$$

Conversely, if the power series (4.5) has a continued fraction expansion of the form (4.6), then the power series (4.3) has a continued fraction expansion of the form (4.2) in which the $b_{p}$ are all equal to zero. We shall call (4.6) an S-fraction since it is the form of continued fraction preferred by Stieltjes.

From the preceding it follows that the condition for (4.5) to have an $S$-fraction expansion (4.6) in which $a_{p} \neq 0, p=0,1,2, \cdots$, is the 
same as the condition for (4.3) to have an expansion (4.2). This condition is that the determinants

$$
c_{0},\left|\begin{array}{ll}
c_{0}, & 0 \\
0, & c_{1}
\end{array}\right|,\left|\begin{array}{ccc}
c_{0}, & 0, & c_{1} \\
0, & c_{1}, & 0 \\
c_{1}, & 0, & c_{2}
\end{array}\right|,\left|\begin{array}{cccc}
c_{0}, & 0, & c_{1}, & 0 \\
0, & c_{1}, & 0, & c_{2} \\
c_{1}, & 0, & c_{2}, & 0 \\
0, & c_{1}, & 0, & c_{2}
\end{array}\right|, \ldots
$$

be different from zero. From this we readily conclude the well known result that the power series (4.5) has an $S$-fraction expansion if and only if the determinants

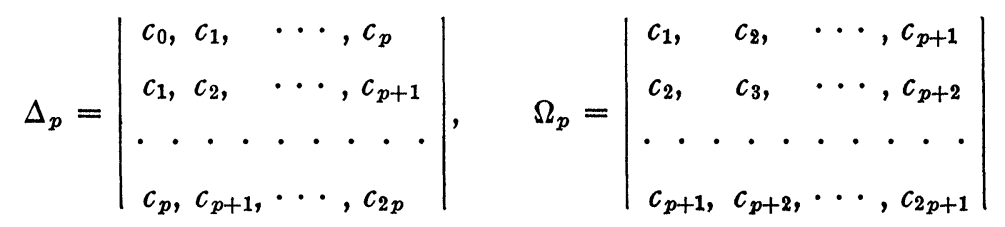

$$
\begin{aligned}
& (p=0,1,2, \cdots)
\end{aligned}
$$

are all different from zero.

It is immediately evident that the algorithm of $\$ 2$ can be used to compute the coefficients in (4.6) if we there replace $c_{2 n}$ by $c_{n}$ and $c_{2 n+1}$ by 0 .

5. A theorem of Stieltjes. A remarkable formulation of the problem of expanding a power series into a continued fraction was given by Stieltjes [3, p. 184]. Rogers [2] rediscovered part of the result of Stieltjes in a slightly different form. We offer the following formulation of the theorem.

The problem of expanding the power series

$$
\frac{1}{z}-\frac{c_{1}}{z^{2}}+\frac{c_{2}}{z^{3}}-\frac{c_{3}}{z^{4}}+\cdots
$$

into a continued fraction

$$
\frac{1}{b_{1}+z}-\frac{a_{1}}{b_{2}+z}-\frac{a_{2}}{b_{3}+z}-\cdots
$$

is equivalent to the problem of securing a power series identity of the form

$$
Q(x+y)=Q(x) Q(y)+a_{1} Q_{1}(x) Q_{1}(y)+a_{1} a_{2} Q_{2}(x) Q_{2}(y)+\cdots,
$$


where $a_{p} \neq 0, p=1,2,3, \cdots$,

$$
Q_{n}(z)=\frac{z^{n}}{n !}+\pi_{n, n+1} \frac{z^{n+1}}{(n+1) !}+\pi_{n, n+2} \frac{z^{n+2}}{(n+2) !}+\cdots,
$$

and

$$
Q_{0}(z)=Q(z)=1+c_{1} \frac{z}{1 !}+c_{2} \frac{z^{2}}{2 !}+c_{3} \frac{z^{3}}{3 !}+\cdots
$$

The formulas connecting the various constants are:

$$
\begin{aligned}
& \pi_{0,0}=1, \quad \pi_{p, q}=0 \text { for } p>q ; \\
& \left(\pi_{0, q}, \pi_{1, q}, \pi_{2, q}, \cdots\right) \\
& =\left(\pi_{0, q-1}, \pi_{1, q-1}, \pi_{2, q-1}, \cdots\right)\left(\begin{array}{cccc}
b_{1}, & 1, & 0, & 0, \ldots \\
a_{1}, & b_{2}, & 1, & 0, \ldots \\
0, & a_{2}, & b_{3}, & 1, \ldots \\
. & . & . & .
\end{array}\right) ; \\
& b_{1}=\pi_{0,1}, \quad b_{p}=\pi_{p-1, p}-\pi_{p-2, p-1}, \quad p=2,3,4, \cdots ; \\
& c_{p+q}=\pi_{0, p} \pi_{0, q}+a_{1} \pi_{1, p} \pi_{1, q}+a_{1} a_{2} \pi_{2, p} \pi_{2, q}+\cdots .
\end{aligned}
$$

This combines the idea of Rogers with a formulation of Stieltjes' algorithm particularly adapted to the $J$-fraction. A part of this is given in [1, pp. 328-329]. We omit the proof.

Both Stieltjes and Rogers gave the example:

$$
\int_{0}^{\infty} \operatorname{sech}^{k} u e^{-z u} d u=\frac{1}{z}+\frac{1 \cdot k}{z}+\frac{2(k+1)}{z}+\frac{3(k+2)}{z}+\ldots .
$$

This can be obtained almost by inspection from the identity

$$
\begin{gathered}
\operatorname{sech}^{k}(x+y)=(\cosh x \cosh y+\sinh x \sinh y)^{-k} . \\
\text { Bibliography }
\end{gathered}
$$

\section{BIBLIOGRAPHY}

1. O. Perron, Die Lehre von den Kettenbrulchen, Leipzig and Berlin, 1929.

2. L. J. Rogers, On the representation of certain asymptotic series as convergent continued fractions, Proc. London Math. Soc. (2) vol. 4 (1907) pp. 72-89.

3. T. J. Stieltjes, Oeuvres, vol. 2.

ILLINOIS INSTITUTE of TeChNOLOGY 